\title{
In situ straining experiments in the transmission electron microscope
}

\section{U. MESSERSCHMIDT}

Max Planck Institute of Microstructure Physics, Weinberg 2, Hall/S. 06120, Germany

In the high voltage electron microscope (HVEM) in situ straining experiments have been performed on a number of metals at room temperature and on solar silicon and silicon nitride at high temperatures. The high-temperature deformation device and some recent results on in situ deformation are described. Details of the interaction between dislocations and ordered precipitates are obtained for Al-Li. In Ti-Al dislocations move in a jerky way between pinned states with bowed out dislocation segments. Dislocation multiplication is triggered by a dislocation reaction in silicon. At high temperature, fracture of silicon nitride is accompanied by crack branching and bridging by long grains.

$\mathbf{1}$

Introduction

In situ experiments in the transmission electron microscope allow the direct observation of the microprocesses of deformation and fracture. However, their interpretation is complicated by thin film effects and, especially in the HVEM, by radiation damage. Recently, a new stage has been designed for deformation at temperatures above $1000^{\circ} \mathrm{C}$. In the present paper, the new stage is described. Some results are presented on the deformation of metals at room temperature and of silicon and silicon nitride at high temperatures. They demonstrate that inspite of their limitations in situ experiments yield results which cannot be obtained by other techniques.

\section{Design of a high-temperature deformation stage for the HVEM}

A number of deformation stages for transmission electron microscopes have been designed which apply resistance heating to reach high temperatures. They are limited to temperatures below about $1000^{\circ} \mathrm{C}$. In a pure heating stage, temperatures far beyond this limit have been attained by electron bombardment of the specimen holder [1]. A new top entry double-tilting stage on this basis for high-temperature straining in the HVEM is outlined in Fig. 1a. It consists of a fixed copper lever (1), which is mounted in the water-cooled tilting cone (2) of the cardanic suspension (3) of the tilting stage. The movable lever (4) is joined to the fixed one by a leaf spring (5). Both levers are inclined with respect to the electron beam. The levers are cooled by water pipes (6) including copper lamellae (7) at the contact plane with the parts carrying the hot specimen grips. The deformation is driven by thermal expansion of the rod (8) carrying a heating coil. The specimen grips (9) are made of W-23Re. Their connections to the copper levers have the shape of double-T bars. While the grips are electrically connected to ground, W coil filaments (10), arranged in U-shaped notches in the specimen grips, and their supporting radiation shields (11) are connected to a negative voltage $(700 \mathrm{~V})$. The electron current between the filaments and the surrounding notches heats the grips and indirectly also the specimen (12). It is connected with the grips by $\mathrm{W}$ pins. The force acting on the sample is measured by four semiconducting 


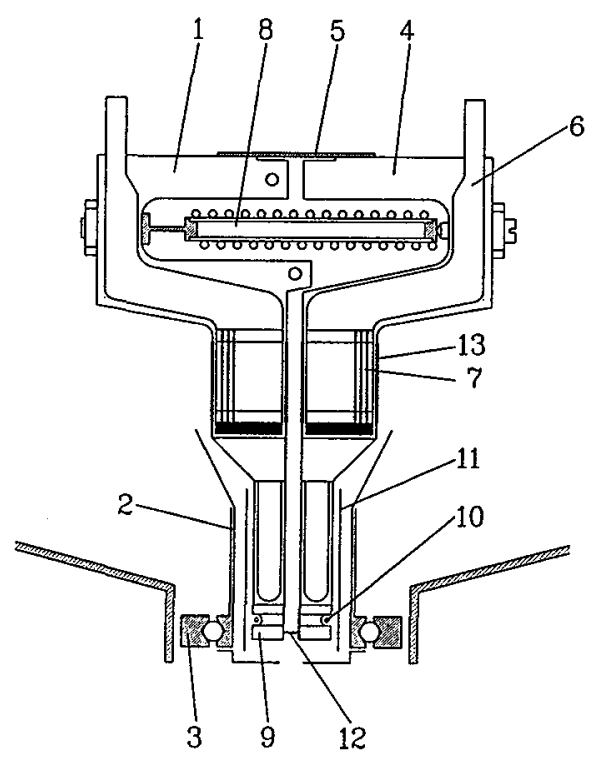

a)

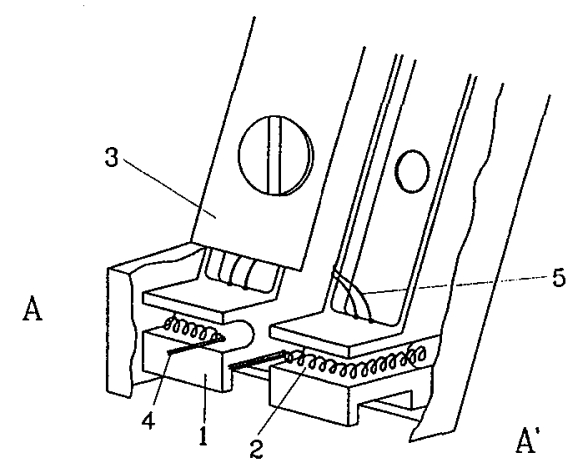

A

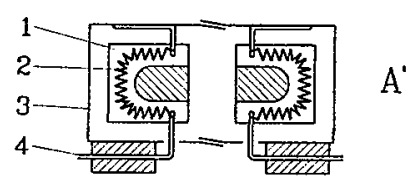

b)

Fig. 1 Outline of double-tilting high-temperature straining stage for the HVEM

strain gauges (13). Fig. 1b shows the hot part of the apparatus with the grips (1) in a perspective view and in a cross section in the plane of the filaments (2). The thermal shields (3) carry also the insulated connections (4) of the filament current. The temperatures of the grips are measured by thermocouples (5). The operation of the stage has been checked by a number of high-temperature straining experiments. Maximum grip temperatures of $1250^{\circ} \mathrm{C}$ have been reached. Unfortunately, the response time of the drive mechanism amounts to several minutes so that the loading rates have to be low. Some experimental results on high-temperature deformation are described in Sections 5 and 6.

\section{Precipitation hardening in aluminium alloys}

$\mathrm{Al}-\mathrm{Zn}-\mathrm{Mg}$ [2], Al-Ag [3] and Al-Li [4, 5] alloys have been deformed in situ in the HVEM at room temperature. All the materials are hardened by precipitates of different phases. The micrographs show the dislocations under load bowing out between the precipitates. The spacings along the dislocations between the precipitates and the curvatures of the bowed-out dislocation segments have been measured and compared with the results of conventional transmission electron microscopy of the precipitates and macroscopic measurements of deformation data. In most cases, the microscopic in situ data differ from results of other methods with respect to the density and strength of the acting obstacles to dislocation motion. The evaluations also allow the investigation of the dependence of the curvature of the dislocation segments on the segment length, as predicted by the line tension theory. This yields a numeric constant in the logarithmic term of the expression of the dislocation line energy and line tension [6]. The experimental value of the logarithmic factor is only about half the theoretical one. This would imply a much lower line energy and line tension of dislocations than usually assumed.

Al-Li alloys are hardened by precipitates of the ordered $\delta$ ' phase. Dislocations in the matrix cutting these precipitates have to form an antiphase boundary (APB) inside the particles. A second dislocation can annihilate the antiphase boundary and is therefore pushed forward. Thus, dislocations should move in pairs for energetic reasons. Fig. 2 shows such pairs which have been pushed out of the plastic zone of a crack. An analysis of the force balance between both dislocations of the pairs [5], using the particle size and spacings, the curvature of the segments as well as the distances between the dislocations of 


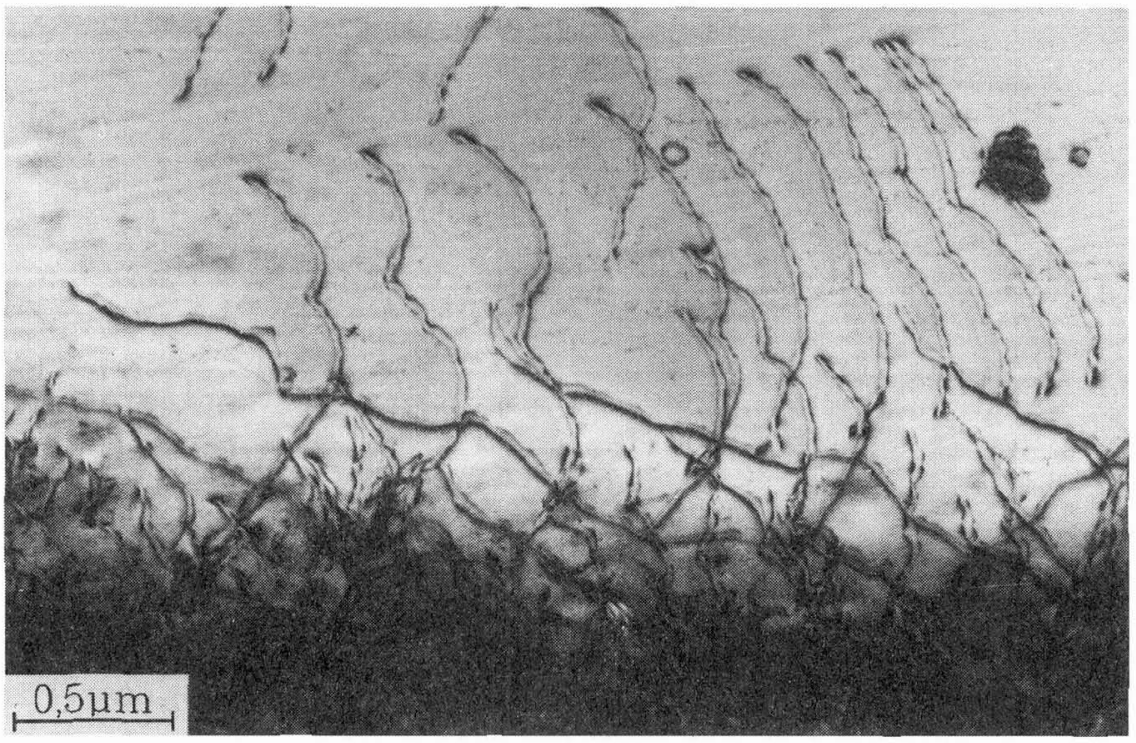

Fig. 2 Dislocation pairs pushed out of the plastic zone of a crack in Al-8.36at\% Li during in situ straining in the HVEM. Accelerating voltage (in all micrographs of the paper) $1000 \mathrm{kV}$

the pairs, is not consistent with the assumption generally made that the precipitates interact with the dislocations solely by order hardening. This is in accord with the observation that many segments of the trailing dislocations bow out in forward direction and not backwards as expected from the simple theory. The discrepancy is explained by the action of a second interaction mechanism, which most probably is modulus hardening. Again, these details in the dislocation mechanisms can only be deduced from a direct observation of the dislocation motion.

\section{Dislocation motion in Ti-AI}

In $\gamma$ phase Ti-A1, dislocations may have Burgers vectors of the type $a / 2<110$ ] or $\mathrm{a} / 2<112$ ], or they are superdislocations with Burgers vectors a $<011]$. The first Burgers vectors are the shortest and these dislocations may undergo a four-fold dissociation including stacking faults. The latter ones dissociate into two dislocations of the type $\mathrm{a} / 2<011$ ] which enclose an APB. The trailing dislocations may additionally dissociate into partials enclosing a superlattice intrinsic stacking fault. The APB energy may be lower on $\{100$ ) planes so that these dislocations may form locked configurations similar to superdislocations in $\mathrm{L}_{2}$ alloys. All dislocations may feel deep Peierls valleys along < 110] (for a review see [7]). It is supposed that at rest all the dislocations get pinned by different processes and then have to be unpinned to get mobile. The details of these processes and the origin of the flow stress anomaly have not been explained satisfactorily. Besides, the pinning/unpinning has not yet been observed experimentally in Ti-Al.

First in situ straining experiments have been carried out on Ti-52at\%Al polycrystals with very large grains at room temperature [8]. Fig. 3 shows mobile dislocations which were generated during in situ deformation. These dislocations have a curled shape similar to materials exhibiting precipitation hardening. Their character has not yet been exactly determined, but they are most probably in screw orientation. From video sequences it is concluded that the dislocations are pinned most of the time and that they move in a jerky way between the pinned states in times shorter than that between video frames. The slip distances usually amount to fractions of a micrometer. An interpretation of the results will be tried after the type of the dislocations is identified. Further studies to investigate the temperature dependence of the dislocation motion are in progress. 


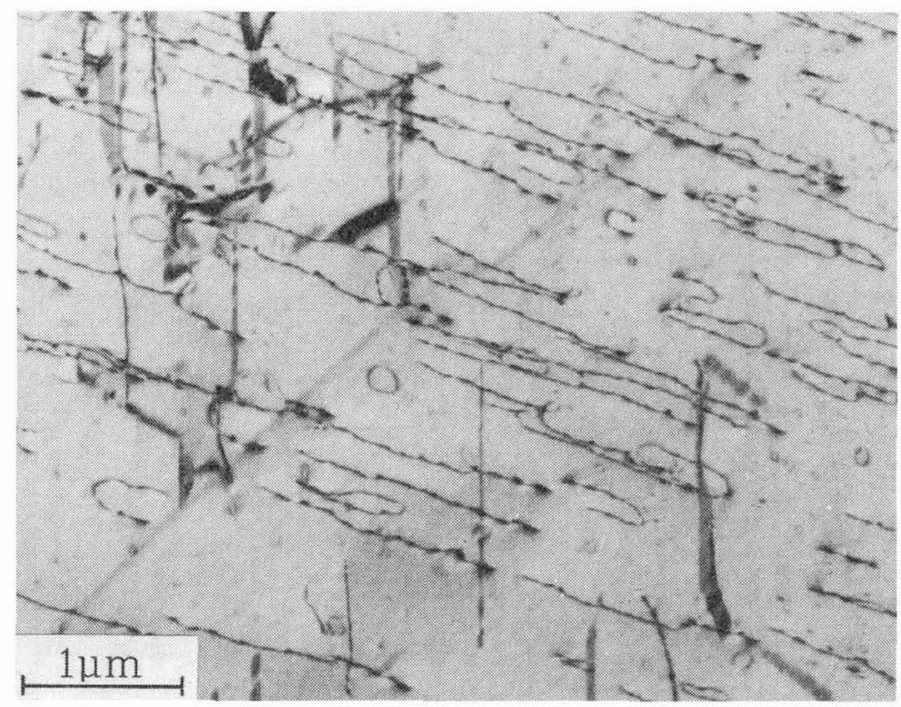

Fig. 3 Moving dislocations in Ti-52at\%Al

Cast solar silicon crystals have been deformed in situ in the temperature range between 780 and $530{ }^{\circ} \mathrm{C}$ in the stage described in Section 2 [9]. The dislocations move quite continuously at all temperatures. Preferential orientation in Peierls valleys is detected at lower temperatures but it is only little pro-
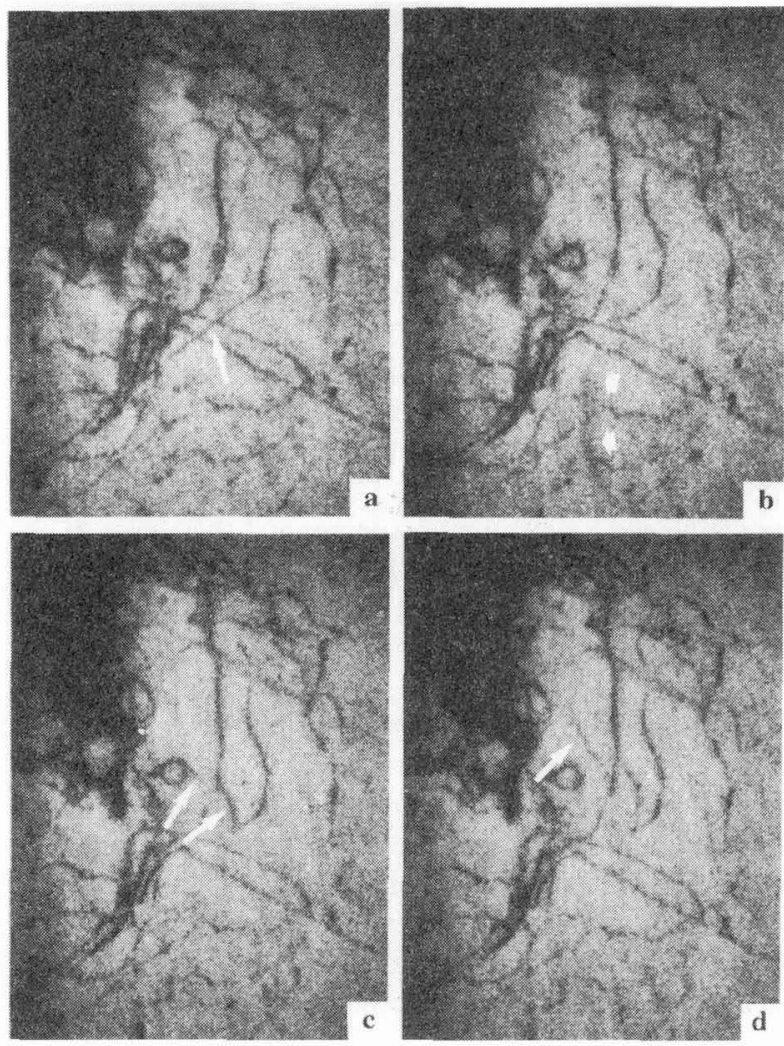

nounced for the moving dislocations.

Dislocation velocities have been determined at low temperature in dependence on the load. They yield a so-called stress exponent between 1 and 2. From the temperature dependence of the load necessary to reach certain dislocation velocities, the activation energy of dislocation motion is estimated to be about $1.5 \mathrm{eV}$. These data are in agreement with macroscopic measurements [10], i.e. the in situ experiments yield reliable data on the dislocation motion. Further evaluations aim at a determination of the effective stresses from dislocation curvatures.

Fig. 4 Sequence of a video recording of the initiation and operation of a dislocation source in solar silicon 
During in situ straining many dislocations are generated from localized dislocation sources. These sources seem to be activated by dislocation reactions, as illustrated by the video sequence in Fig. 4 and a schematical drawing of the possible geometry in Fig. 5. The initial situation is shown in Fig. 4a. The dislocation labelled by an arrow (on plane 1 in Fig. 5a) is met by another one (not visible in Fig. 4 but on the video tape) approaching from below on a different slip plane (plane 2 in Fig. 5a). If both dislocations have the same Burgers vector parallel to the intersection line between the slip planes, one part of the dislocation in plane 1 can combine with a part of the dislocation in plane 2 and the remaining parts can do the same as depicted in Fig. 5b. Afterwards, e.g. the right dislocation may move out of the crystal and the left branch may start to rotate in plane 2 around the end point of the branch in plane 1 , as shown in Figs. $5 \mathrm{c}$ and $4 \mathrm{~b}$ to $\mathrm{d}$. The dislocation source was observed to generate a number of new dislocations. Similar sources are activated at grown-in dislocations in dislocation walls. In contrast to other materials, these localized sources are the main origin of new dislocations during the plastic deformation of silicon.
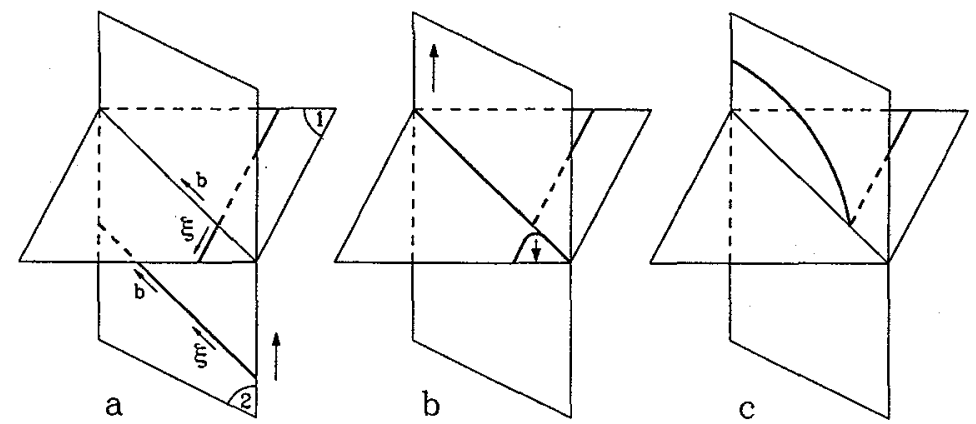

Fig. 5 Schematic drawing of the activation of a dislocation source

A few in situ fracture experiments have been performed on silicon nitride at a temperature of about $1150^{\circ} \mathrm{C}[11]$. The material has small but elongated grains connected by a grain boundary phase. At room temperature intergranular fracture dominates but long grains bridging the crack are cleaved. At high temperature the crack branches as shown in Fig. 6a. The cracks are only scarcely opened. In front of the cracks small microcracks grow at large grains as shown in Fig. 6b, i.e. these grains are pulled out along the grain boundary phase. Crack branching and bridging by elongated grains result in the increase of the fracture energy at high temperatures.

\section{Discussion}

In situ experiments in the transmission electron microscope are well suited to investigate the microprocesses of plasticity and fracture. This holds especially true of dislocation dynamics and the influence of the mechanisms controlling the dislocation mobility. A serious problem is the radiation damage created in almost all materials in the HVEM. For many materials radiation damage can be avoided by using beam voltages of $200 \mathrm{kV}$, or below. In this case, however, the transmittable foil thickness is very small so that the radius curvature of bowed-out dislocation segments or the distances between the pinning points are not small enough with respect to the foil thickness. These features are particularly important for the investigation of the dislocation motion in intermetallic alloys as described in Section 4. The pinning of dislocations can realistically be observed only in sufficiently thick specimens. This requires high beam voltages with the danger of radiation damage, which may cause additional pinning effects. Further experiments are necessary to elucidate the situation. Nevertheless, important information has already been obtained on a number of materials, which would have been difficult to deduce from indirect experiments. 

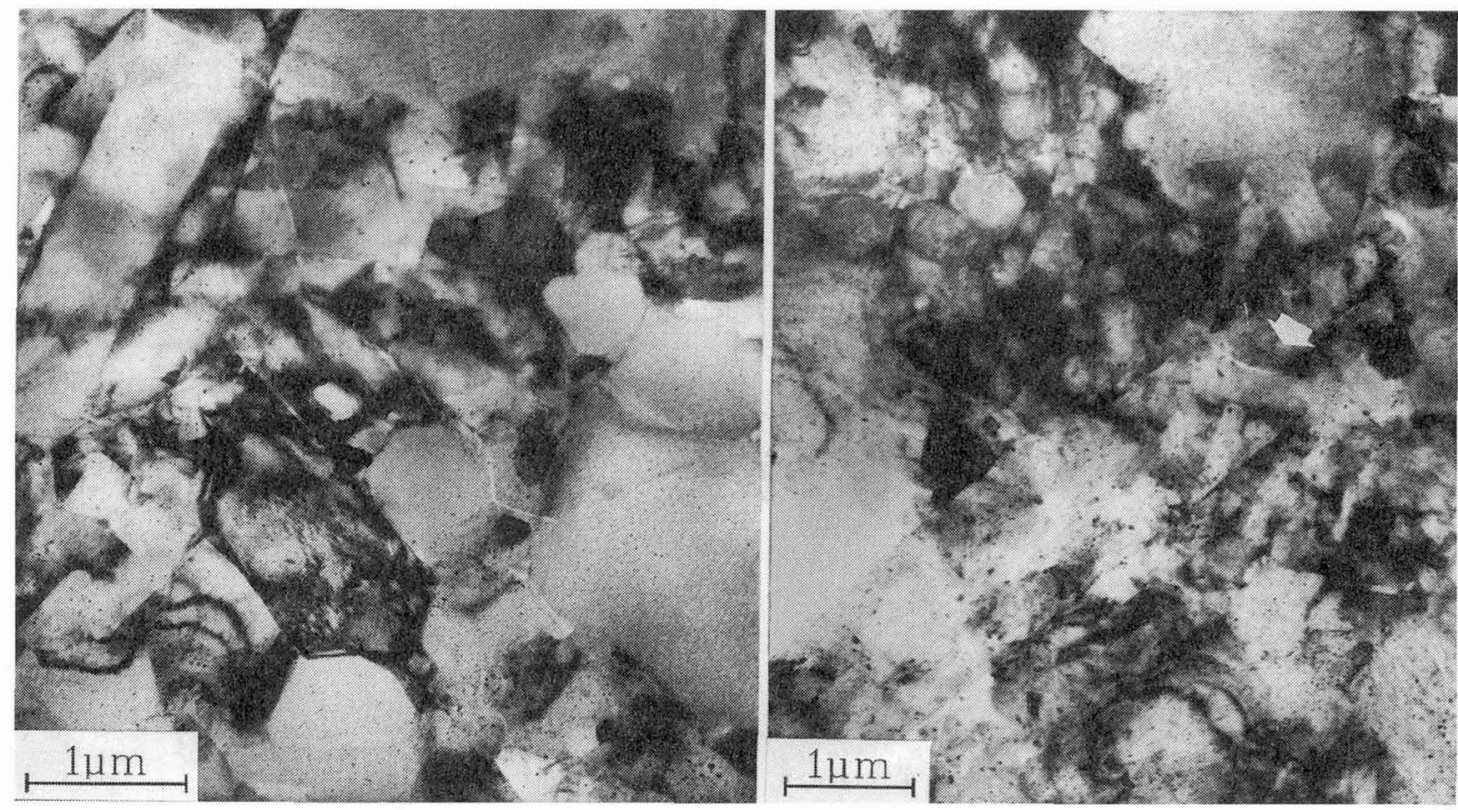

Fig. 6 Cracks in silicon nitride generated during in situ deformation at $1150^{\circ} \mathrm{C}$

\section{Acknowledgements}

The author is very grateful to Drs. Martina Werner, M. Bartsch, D. Baither, and R. Hattenhauer for their cooperation in the experiments. The work on $\mathrm{Ti}-\mathrm{Al}$ is supported by the Deutsche Forschungsgemeinschaft.

\section{References}

1 FUJTA H. and KOMATSU M., Proc. 7th Int. Conf. on High Voltage Electron Microscopy, Berkeley Labs., Berkeley 1983, 371.

2 MESSERSCHMIDT U., APPEL F., BARTSCH M., and GERLACH R., Phys. Status Solidi A 78 (1983) 93.

3 MESSERSCHMIDT U., BARTSCH M., HAASEN P., WILBRANDT P.-J., HORY R., and HATTENHAUER R., to be published

4 NEMBACH E., MESSERSCHMIDT U., KOLBE M., SCHLESIER C., and BARTSCH M., In: Strength of Metals and Alloys, D.G. Brandon et al. (Eds.), Freund Publ., London 1991, 1087.

5 MESSERSCHMIDT U. and BARTSCH M., to be published in Mater. Sci. Eng. A (1993)

6 MESSERSCHMIDT U., to be published in Z. Materialkunde (1993)

7 YAMAGUCHI M. and UMAKOSHI Y., Progr. in Mater. Sci., Vol. 34, W. Christian et al. (Eds.) (1990) 1.

8 HATTENHAUER R., MESSERSCHMIDT U., BARTSCH M., AINDOW M., and JONES I.P., to be published

9 WERNER MARTINA, BARTSCH M. and MESSERSCHMIDT U., to be published

10 ALEXANDER H., In: Dislocations in Solids, F.R.N. Nabarro (Ed.), Vol. 7, North-Holland, Amsterdam 1986, 113.

11 BARTSCH M., MESSERSCHMIDT U. and BAITHER D., to be published 